\title{
Entretien autour de Hamlet
}

Jean-Michel Déprats et Gérard Desarthe

\section{(2) OpenEdition \\ Journals}

Édition électronique

URL : http://journals.openedition.org/shakespeare/1222

DOI : 10.4000/shakespeare.1222

ISSN : 2271-6424

Éditeur

Société Française Shakespeare

Édition imprimée

Date de publication : 1 novembre 1990

Pagination : 157-160

Référence électronique

Jean-Michel Déprats et Gérard Desarthe, "Entretien autour de Hamlet », Actes des congrès de la Société française Shakespeare [En ligne], 8 | 1990, mis en ligne le 01 janvier 2007, consulté le 25 avril 2019. URL : http://journals.openedition.org/shakespeare/1222 ; DOI : 10.4000/shakespeare.1222 


\section{SHAKESPEARE \\ ET LA GUERRE}

Ouvrage publié avec le concours de la Commission

des Communautés Européennes 
Illustration de la couverture : DERRICKE'S Image of Ireland, 1586 


\section{Entretien de Jean-Michel Déprats avec Gérard Desarthe}

Hamlet, un enfant qui n'arrête pas de grandir

Ce spectacle est né de ton désir. Tu rêvais depuis toujours d'interpréter Hamlet. Était-ce par envie d'affronter le rôle le plus joué du répertoire shakespearien ou par une attirance plus spécifique pour des aspects du personnage proches de ta sensibilité d'acteur, son inquiétude, sa révolte, sa soif d'absolu par exemple?

Pour tout acteur la confrontation avec Hamlet est un défi qu'il faut relever. Hamlet est la plus grande tragédie populaire, la pièce la plus connue: tout acteur a rendezvous avec Hamlet. Shakespeare est déjà un des dramaturges qui me bouleversent, me touchent, m'agressent, me questionnent le plus. Cette fascination est d'autant plus forte lorsqu'il s'agit d'Hamlet qui restera dans l'histoire théâtrale universelle énigmatique. Le personnage m'a toujours secrètement parlé, intrigué. Cette grande tragédie du doute est une pièce profondément archaïque, brute. Et je trouve que ce théâtre anglais du $17^{\mathrm{e}}$, brutal, sans psychologie, est encore capable de faire la nique au cinéma, à la télévision.

Tu définis Hamlet comme une tragédie du doute, appréciation qui va souvent de pair avec une mise en relief du thème de la temporisation et qui tend à faire d' Hamlet un intellectuel hésitant. Une autre tradition critique et scénique - je pense à Vyssotski ${ }^{I}$ - fait plutôt d'Hamlet un "personnage combattant», qui se heurte à des obstacles mais qui lutte sans cesse. 
Il y a les deux. C'est une pièce sur ce que les critiques appellent la «procrastination», mais l'interprétation romantique fait d'Hamlet un personnage trop rétrospectif, presque lunaire. Or, il est tout le temps en action et ma nature d'acteur m'a poussé à le rendre toujours actif, toujours sur le qui-vive. Pour moi, dès que le spectre le charge de cette mission terrible, il cesse d'être l'intellectuel, l'étudiant de Wittenberg. Son corps, ses nerfs, ses muscles entrent en jeu et il y a une sorte de court-circuit, de collision entre l'esprit et le corps. C'est un personnage qui va jusqu'au bout. C'est un actif: le texte rappelle qu'il s'entraîne régulièrement à l'escrime. Par ailleurs, c'est aussi un personnage très réflexif, que ses doutes, ses interrogations arrêtent périodiquement. J'ai eu envie de montrer un jeune homme totalement pris dans ses contradictions.

Les facteurs de paralysie d'Hamlet sont-ils tous d'origine inconsciente? Dans "What happens in Hamlet ${ }^{2}$, Dover Wilson rappelle les croyances et les débats théologiques de l'époque élisabéthaine sur la nature et l'origine des spectres. Référées à cet univers mental, les interrogations d'Hamlet pendant trois actes sont de vraies questions?

Tout à fait. Il m'a semblé très vite important d'établir dans mon jeu la crédibilité du fantôme. l'apparition du spectre est un point d'accrochage essentiel pour le personnage. C'est une chose souvent éludée parce qu'aujourd'hui on ne croit plus aux fantômes. La belle idée de Patrice Chéreau est d'avoir inventé cette image poétique forte, violente, de Cavalier de la Mort, de Messager de l'au-delà qui vient demander vengeance. Pour moi, c'est aussi le Père qui vient punir. Cette relation qui s'instaure avec Hamlet est trouble. Le spectre dit qu'il fut «moissonné en la fleur de ses péchés», qu'il est au Purgatoire. Pendant tout ce temps, Hamlet pense qu'il a affaire à un démon. En tout cas, cette rencontre le terrasse, c'est un véritable cataclysme mental. 
Autre facteur de trouble lié aux mentalités élisabéthaines: le remariage de Gertrude avec le frère de sonépoux qui était perçu à l'époque comme une relation incestueuse

L'adultère de sa mère est une chose qui le mine, qui le dévore, qu'il ressent comme une blessure dans sa chair. Il le dit très violemment. Le puritanisme du personnage, son rapport nauséeux, problêmatique, à la sexualité et aux femmes est une chose difficile à assumer pour un acteur mais qui est déterminante pour le personnage. L'horreur du comportement de sa mère gangrène de proche en proche son rapport à la femme. La scène avec Ophélie est une scène terrible de sacrifice, d'autodestruction; il l'aime mais lui dit qu'il ne peut pas et ne veut pas l'aimer parce qu'il connaît trop la pourriture des cœurs et des sens.

Y a-t-il des aspects du rôle que tu as réellement découverts en le travaillant?

Je pressentais, plus que je ne comprenais, un certain nombre de choses que le travail confirme; le fait qu'Hamlet a un côté enfant qui n'arrête pas de grandir, qui a tout compris et qui ne s'en sortira pas; le fait que le rôle est une longue réflexion sur la mort. Ça commence dès sa première apparition en noir à la cour et ça parcourt la pièce jusqu'â l'acte cinq où le personnage parvient à en parler de façon détachée, distancée. Devant le fossoyeur, Hamlet a perdu toute son arrogance, il n'a plus la superbe acuité, la finesse, l'humour qu'il avait avant. Pourquoi Shakespeare le fait-il revenir par le cimetière? Je crois qu'il revient sur les traces de son père mort, qu'il cherche la mort, sa mort, plus qu'il ne cherche à se venger.

Dans ton interprétation il rencontra sa mort après un duel étonnant de vigueur, d'énergie physique. Cette énergie, c'est une constante de ton jeu. Ce qui m'a le plus ébloui dans ton travail c'est l'état de totale invention dans lequel tu es en permanence, et l'extraordinaire mobilité de l'esprit et du corps d'un Hamlet fébrile, frénétique, constamment électrisé par la multiplicité des pensées, des pulsions, des désirs qui l'assaillent. 
Il a sans arrêt des impulsions contraires, des élans, des oublis. Il s'échauffe tout le temps. C'est lui qui lance les acteurs dans la tirade sur Pyrrhus puis il oublie le texte, les acteurs le reprennent, il est fasciné, se dit «oui il faut aller jusqu'à cette incandescence de l'action, du verbe, du théâtre» et quelques secondes plus tard, il proclame que cette intensité-là se déploie pour rien, pour Hécube... Et puis ça se renverse, le monologue se termine par une magnifique profession de foi dans le théâtre.

Pour lui, le théâtre est ambivalent, c'est un jeu de masques et de leurres, mais aussi un extraordinaire instrument de vérité.

Il y a les deux bien sûr. Tout est faux, tout est vrai. Tout est masque et tromperie. Pour lui le monde est une gigantesque comédie du paraître et, à part Horatio, tous ont des masques qu'il faut arracher. Le théâtre sert aussi à cela. À dévoiler les visages. À traquer la vérité.

La richesse d'expériences spirituelles, morales, intellectuelles qui fait le personnage implique-t-elle pour ton parcours d'acteur un travail analogue à celui que tu avais fait sur Peer Gynt?

Je vois surtout les différences. Peer Gynt est un voyage initiatique. Hamlet me fait penser aux chromosomes, à une cellule. Tout est en puissance et tout est mort. C'est un personnage nucléaire. On peut essayer de percer le mystère mais ça restera toujours énigmatique, toujours sublime.

\section{NOTES}

(1) Interprète du rôle dans la mise en scène de Lioubimov pour le théâtre de la

(2) Traduit par Dominique Goy-Blanquet sous le titre Vous avez dit Hamlet?, Paris, Aubier. Thêâtre des Amandiers, 1988. 